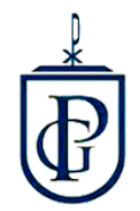

Veritas Et Scientia

Vol. $7, N^{\circ} 1,836-847$

Enero - Junio del 2018.

ISSN $2307-5139$

\title{
ANÁLISIS DE UN MODELO ANALÍTICO PARA EVALUAR LA INTERACCIÓN ENTRE UNA MESA VIBRATORIA Y UNA ESTRUCTURA DE ENSAYO DURANTE ENSAYOS DE SIMULACIÓN SÍSMICA
}

ANALYSIS OF AN ANALYTICAL MODEL TO EVALUATE THE INTERACTION BETWEEN A VIBRATORY TABLE AND AN ESSAY STRUCTURE, DURING SEISMIC SIMULATION TESTS

Carmen Patiño Mendoza ${ }^{1}$

Presentado: 10/02/2017

Aceptado: $15 / 05 / 2018$

Publicado online: 11/06/2018

\section{RESUMEN}

En el presente trabajo de investigación, se analiza un modelo analítico que evalúa la interacción entre mesa vibratoria y estructuras de ensayo, durante ensayos de simulación sísmica. El modelo en estudio es el propuesto por Esparza (1986), que representa la implementación de un controlador por retroalimentación de fuerzas y desplazamiento, éste con la finalidad de ampliar el estudio en mesas vibratorias que se constituyen en herramientas cruciales para analizar el desempeño de estructuras ante acciones sísmicas. A partir de una extensa revisión bibliográfica, en este trabajo de investigación se describen y discuten algunos de los sistemas de control más utilizados, y su efecto en la reproducción de la señal sísmica en mesas vibratorias y para el caso de la estructura de ensayo se ha optado por utilizar una estructura con propiedades de concreto celular, con la finalidad de validar su comportamiento ante eventos sísmicos, como alternativa de construcción eficiente e identificar sus parámetros dinámicos.

Palabras clave: sistema de control, ensayo dinámico, mesa vibratoria, simulador sísmico.

\footnotetext{
${ }^{1}$ Magíster en ingeniería civil, con mención en estructuras
} 


\begin{abstract}
In the present research work, an analytical model that evaluates the interaction between vibratory table and test structures, during seismic simulation tests is analyzed. The model under study is the one proposed by Esparza (1986), which represents the implementation of a controller by feedback of forces and displacement, with the purpose of expanding the study in vibratory tables that constitute crucial tools to analyze the performance of structures before seismic actions. It should be noted that these devices have become a fundamental part in the field of earthquake-resistant engineering, allowing us to study complex structural dynamical models. Based on an extensive literature review, this research paper describes and discusses some of the most commonly used control systems and their effect on the reproduction of the seismic signal on vibrating tables and, in the case of the test structure, opted to use a structure with cellular concrete properties, in order to validate its behavior against seismic events, as an efficient construction alternative and identify its dynamic parameters.
\end{abstract}

Key words: control system, dynamic test, vibratory table, seismic simulator.

\title{
INTRODUCCIÓN
}

El presente estudio analiza un modelo analítico lineal propuesto por Esparza en 1986, para simuladores sísmicos electrohidráulico de un grado de libertad, considerando los aspectos más relevantes como son: el control de desplazamientos y compensación del sistema, con lo cual se pretende evaluar la interacción entre la mesa vibratoria y una estructura de ensayo con características de concreto celular, para finalmente, encontrar valores característicos que permitan describir el comportamiento dinámico del sistema propuesto, durante los ensayos de simulación sísmica. Según Esparza(1986), se estudia el efecto de colocar una estructura de ensayo de dos grados de libertad de concreto celular acoplada a la plataforma del simulador, apoyándonos en la herramienta MATLAB- Simulink, con la representación por diagrama de bloques. Reproduciéndose la señal sísmica, con las características del actuador, para evaluar la interacción de las propiedades dinámicas del sistema, evaluando el error relativo - RMS que se espera alcance valores menores al $10 \%$. Con la herramienta de Simulink se permite confeccionar un ambiente de trabajo gráfico dentro de MATLAB, a partir de diagramas de bloques. Además, soporta simulación, generación automática de código, así como pruebas continuas para verificar el buen funcionamiento de sistemas. Con ellos se logró analizar los parámetros representativos del espécimen de ensayo, que para el presente trabajo de investigación adoptará las propiedades más resaltantes del concreto celular, material escogido por sus características, para enfatizar su uso en la construcción de edificaciones, que han sido incluidos en el modelo analítico de simulación sísmica. Lo anteriormente indicado, nos permite evaluar la interacción dinámica entre la estructura y la mesa vibratoria, cuando el modelo es sometido a un registro de aceleraciones sísmicas, comparando los resultados teóricos y experimentales. Asimismo, como experiencia se plantea evaluar la interacción entre una mesa vibratoria con una estructura de ensayo con características de concreto celular, porque consideramos que el aporte de concretos livianos en su mayoría permiten una simulación más específica 
con fines académicos, el interés de enfatizar en el diseño de un modelo analítico para evaluar la interacción entre una mesa vibratoria y una estructura de ensayo durante ensayos de simulación sísmica, nos permite integrar a estructuras con características definidas como es el caso del concreto celular, se escoge este material por sus propiedades principales como su resistencia a la compresión, el módulo de elasticidad, la resistencia al fuego, su aislamiento térmico, y otras propiedades que nos hacen suponer que es un material que brindará mejor comportamiento ante solicitaciones sísmicas, y nuestra región Tacna, se encuentra en un silencio sísmico que nos obliga a edificar bajo consideraciones extremas de un movimiento sísmico, debido a que después del sismo del año 2001, las viviendas de concreto armado presentaron daños y el peligro de un nuevo terremoto en la región del Sur del Perú, está latente, debido a que se sabía que el terremoto ocurrido el 23 de junio del 2001, no cumplía las condiciones para ser considerado repetitivo, del gran terremoto que se produjo en esta región el 13 de agosto de 1868.

En Inglaterra, T.Y. Yang et al, (2017) plantea un modelo donde el modelo propuesto de control jerárquico puede mejorar el rendimiento de seguimiento de aceleración en comparación con el controlador de acción proporcional - integral - derivativa (PID). En Colombia, Bernal, Aponte y Carrillo (2015) refieren que La importancia de las mesas vibratorias se evidencia por su contribución a la ingeniería sismorresistente en el último siglo. En Estados Unidos, Blondet y Esparza (1988), presenta un modelo analítico para evaluar las características de desempeño de los simuladores sísmicos unidireccionales, con la finalidad de explicar la degradación del rendimiento durante las pruebas de simulación sísmica con estructuras muy pesadas, lo cual nos permite establecer pautas para el diseño de sistemas de mesas de vibración más confiables. En Lima, Blondet y Velásquez (2016), refieren que "los efectos de la interacción entre la estructura y la mesa vibratoria se manifiestan con la distorsión peaknotch (resonancia-antiresonancia) en la respuesta en el dominio de la frecuencia. La máxima amplificación ocurre a la frecuencia natural de la estructura de ensayo. El sistema de control no elimina la degradación en la respuesta causada por los efectos de la interacción mesa-estructura" Base teórico científicas.

Los Sistemas de control estructural, surge como una alternativa al diseño sismorresistente convencional, que combina adecuadamente la energía elástica y disipación en sus elementos principales. Bonilla (2012), refiere que lo que se busca es la modificación de las propiedades dinámicas del edificio, de forma que éste reduzca su energía de entrada o evite actitudes resonantes, la disipación de energía y el control con dispositivos que ejerzan fuerzas que contrarresten la acción Sísmica. Para Ricardo Hernández Gaviño (2010), un sistema de control automático es una interconexión de elementos que forman una configuración denominada sistema, de tal manera que el arreglo resultante es capaz de controlar se por sí mismo. Para Richard C. Dorf (1983), Yasundo Takahashi, Michael J. Rabins y David M. Auslander (1970) y Howard L. Harrison \& John G. Bollinger (1983), definen que existen dos maneras básicas de concebir un proceso de control: estableciendo un lazo abierto o estableciendo un lazo cerrado. Respecto a la clasificación de los sistemas de control, Hernández G. (2010); los clasifica en sistemas de lazo abierto (o no automáticos) y sistemas de lazo cerrado (retroalimentados o automáticos). Esparza (1986), nos plantea que, para un mismo problema de control, o un mismo sistema, de ser concebido en lazo abierto o en lazo cerrado, dependerá de las componentes o partes del sistema que son objeto del control. Asimismo, para Esparza (1986), los diagramas de bloques son representaciones esquemáticas que permiten visualizar las funciones realizadas por cada elemento o componente del sistema. Utilizando los diagramas de bloques se puede evidencias específicamente la contribución de cada componente en el global del trabajo dinámico del sistema. Para Takahashi, et al (1970) y E.P. Popov (1962) y Ogata (1974), es importante resaltar que un diagrama de bloques contiene información sobre el desempeño dinámico del sistema, pero no sobre la constitución física del mismo. Un mismo diagrama de bloques puede representar sistemas de diversas naturalezas.

Existen los modelos de oscilador viscoelástico de un grado de libertad y el Modelo analítico con efectos de interacción que presenta una estructura de $\mathrm{N}$ grados de libertad sobre una mesa vibratoria 


\section{OBJETIVOS}

- Analizar los componentes de las mesas electrohidráulicas con movimiento en 1 grado de libertad.

- Analizar el modelo durante la reproducción de señales armónicas y sísmicas, para una mesa electrohidráulica uniaxial sin estructura.

- Analizar el modelo durante la reproducción de señales armónicas y sísmicas, para una mesa electrohidráulica uniaxial cargada con estructura típica de concreto celular.

- Identificar los parámetros dinámicos de la estructura de concreto celular acoplada en la mesa vibratoria uniaxial.

\section{METOdOlOgÍA}

Investigación aplicada y exploratoria, de diseño descriptivo y correlacional en el ámbito de la investigación de ensayos de simulación sísmica y sistemas de control.Se está representando una estructura de ensayo con comportamiento elástico y lineal de concreto celular cuyas unidades de estudio serán cuantitativas de tipo discretas; para diferentes escenarios donde intervienen los ensayos de simulación sísmica, representados en la interacción con una mesa vibratoria electrohidráulica con 1 grado de libertad: horizontal. La técnica de recolección de los datos fue mediante la observación durante los ensayos de simulación sísmica, analizando la interacción que presenta la mesa vibratoria y una estructura de ensayo, y trabajo en gabinete para el análisis del modelo analítico y sistema de control, propuesto por Esparza en 1986. Se confeccionó hojas de cálculo para registrar los datos en el entorno de MATLAB - Simulink. Los resultados de la representación del modelo con una estructura de 02 grados de libertad, determinaron la masa, amortiguamiento y rigidez con los cuales se analizaron el modelo analítico, comparando los resultados experimentales versus calculados.

El análisis se realizó primero para cargas armónicas controlables, luego se analizó para señales sísmicas registradas, demostrando con esto la amplificación que puede llegar alcanzar la interacción de la estructura con mesa; asimismo, se determinó el error relativo con RMS para las señales sísmicas, finalmente, se representa la correspondencia de la mesa propuesta por Esparza (1986) con el modelo implementado en MATLAB -Simulink al que se le adicionó ruido y señales sísmicas.

\section{RESULTADOS}

\section{CÁLCULO DEL ERROR RELATIVO}

La medida relativa del error relativo mediante la Raíz Media Cuadrática (RMS) se usa para comparar la referencia de amplitud con la realimentación de la señal.

El resultado, es un error relativo del $17.8 \%$, cuando a frecuencia es $5 \mathrm{~Hz}$, para un sistema actuador mesa - estructura de 1 grado de libertad. Sometido a simulación por carga armónica.

Con esta aplicación, se determinará el error relativo que se presenta para frecuencias de $5 \mathrm{~Hz}$, $7.5 \mathrm{~Hz}, 10 \mathrm{~Hz}, 12.5 \mathrm{~Hz}, 15 \mathrm{~Hz}, 17.5 \mathrm{~Hz}, 20 \mathrm{~Hz}, 22.5 \mathrm{~Hz}$ y $25 \mathrm{~Hz}$, con amplitudes de la señal de comando de $\mathrm{X}_{0}=0.1 \mathrm{~m}, 0.2 \mathrm{~m}, 0.3 \mathrm{~m}, 0.4 \mathrm{~m}$. 
Tabla 1: Resultados de la simulación, para calcular el error relativo

\begin{tabular}{|c|c|c|c|c|c|}
\hline $\mathrm{N}^{\circ}$ & $X_{p}(5 \mathrm{~Hz})$ & $X_{c}(5 \mathrm{hz})$ & $\left(X_{p}-X_{c}\right)$ & $\left(X_{p}-X_{c}\right)^{2}$ & $X_{p}{ }^{2}$ \\
\hline 1 & 0.000000 & 0.000000 & 0.000000 & 0.000000 & 0 \\
\hline 2 & 0.005000 & 0.003100 & 0.001900 & 0.000004 & 0.000025 \\
\hline 3 & 0.010000 & 0.006300 & 0.003700 & 0.000014 & 0.0001 \\
\hline 4 & 0.010000 & 0.009400 & 0.000600 & 0.000000 & 0.0001 \\
\hline 5 & 0.012000 & 0.012500 & -0.000500 & 0.000000 & 0.000144 \\
\hline 6 & 0.026000 & 0.015600 & 0.010400 & 0.000108 & 0.000676 \\
\hline 7 & 0.038000 & 0.018700 & 0.019300 & 0.000372 & 0.001444 \\
\hline 8 & 0.019600 & 0.021800 & -0.002200 & 0.000005 & 0.00038416 \\
\hline 9 & 0.049000 & 0.045000 & 0.004000 & 0.000016 & 0.002401 \\
\hline 10 & 0.073000 & 0.060000 & 0.013000 & 0.000169 & 0.005329 \\
\hline 11 & 0.096000 & 0.080000 & 0.016000 & 0.000256 & 0.009216 \\
\hline 12 & 0.100000 & 0.100000 & 0.000000 & 0.000000 & 0.01 \\
\cline { 5 - 7 } & & $\Sigma=$ & 0.066200 & 0.000944 & 0.029819 \\
\cline { 4 - 6 } & & & &
\end{tabular}

En la tabla 2, se presenta los resultados obtenidos del estudio del error relativo medido con la raíz media cuadrática (RMS), para determinar la fidelidad en la reproducción de la señal de comando $\left(X_{c}\right)$ y la respuesta de la plataforma $\left(X_{p}\right)$, se tomó los datos para frecuencias de $5 \mathrm{~Hz}, 7.5 \mathrm{~Hz}$ y $10 \mathrm{~Hz}$, con entrada de amplitudes para la señal de comando $\left(x_{0}\right)$ de $0.1 \mathrm{~m}, 0.2 \mathrm{~m}, 0.3 \mathrm{~m}, 0.4 \mathrm{~m}$ y $0.5 \mathrm{~m}$. En frecuencia de $5 \mathrm{~Hz}$, el error relativo es casi de $100 \%$, por eso en esta zona la fidelidad en la reproducción de la señal de comando, presenta antiresonancia y es influencia directamente por la frecuencia excitadora del sistema. En frecuencias de $7.5 \mathrm{~Hz}$, el sistema presenta una correspondencia en relación con la amplitud de la señal de comando $\left(\mathrm{x}_{0}\right)$, presentando un error relativo del orden de $50 \%$, efectivamente esta tendencia, se evidencia cuando el sistema es sometido a carga armónica para mesa vacía y cargada, la correspondencia en la fidelidad de reproducción de la señal, es casi del orden de $50 \%$, en decrecimiento. En frecuencias de $10 \mathrm{~Hz}$, se presenta situación similar, el error relativo es de aproximadamente $50 \%$, esta relación de la amplificación y efectividad de la reproducción de la señal.

Tabla 2: Resultados del error relativo para frecuencias de $5 \mathrm{~Hz}, 7.5 \mathrm{~Hz}$ y $10 \mathrm{~Hz}$.

\begin{tabular}{|c|c|c|c|}
\hline $\mathbf{X o}(\mathbf{m})$ & $\mathbf{f = 5} \mathbf{~ H z}$ & $\mathbf{f = 7 . 5} \mathbf{~ H z}$ & $\mathbf{f = 1 0} \mathbf{~ H z}$ \\
\hline $\mathbf{0 . 1}$ & $92 \%$ & $45 \%$ & $53 \%$ \\
\hline $\mathbf{0 . 2}$ & $97 \%$ & $51 \%$ & $48 \%$ \\
\hline $\mathbf{0 . 3}$ & $98 \%$ & $52 \%$ & $46 \%$ \\
\hline $\mathbf{0 . 4}$ & $99 \%$ & $53 \%$ & $45 \%$ \\
\hline $\mathbf{0 . 5}$ & $100 \%$ & $54 \%$ & $45 \%$ \\
\hline
\end{tabular}




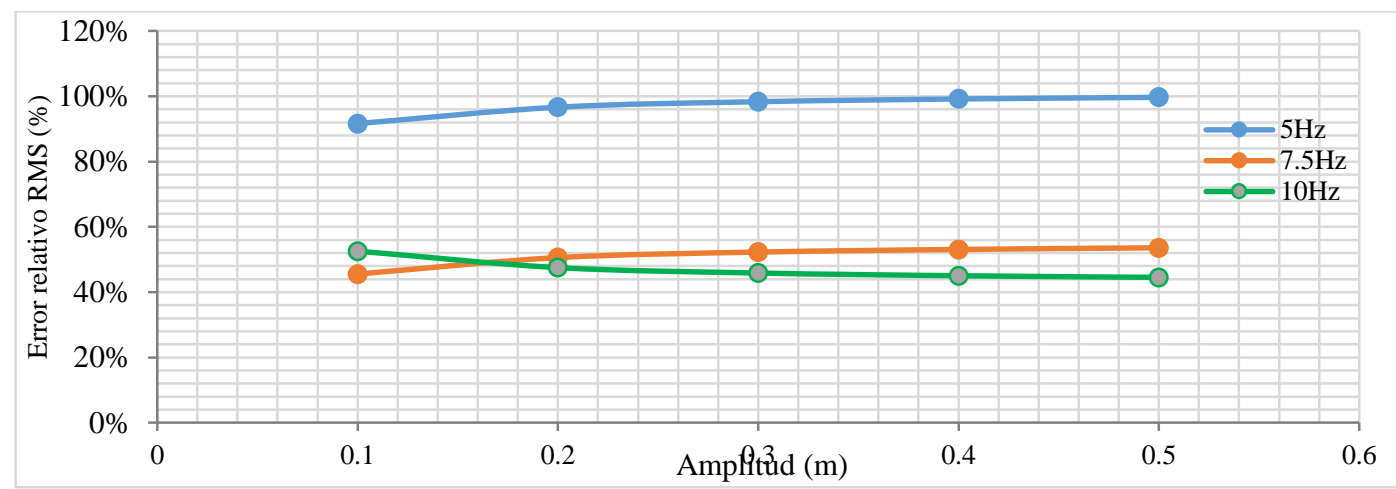

Figura 1- Error relativo para $\mathrm{f}=5 \mathrm{~Hz}, 7.5 \mathrm{~Hz}$ y $10 \mathrm{~Hz}$, para amplitudes de $0-0.5 \mathrm{~m}$.

Fuente: Implementación en MATLAB - Simulink

En la tabla 3, se presenta los resultados obtenidos del estudio del error relativo medido con la raíz media cuadrática (RMS), para determinar la fidelidad en la reproducción de la señal de comando $\left(X_{c}\right)$ y la respuesta de la plataforma $\left(X_{\mathrm{p}}\right)$, se tomó los datos para frecuencias de $12.5 \mathrm{~Hz}, 15 \mathrm{~Hz}$ y $17.5 \mathrm{~Hz}$, con entrada de amplitudes para la señal de comando $\left(x_{0}\right)$ de $0.1 \mathrm{~m}, 0.2 \mathrm{~m}, 0.3 \mathrm{~m}, 0.4 \mathrm{~m}$ y $0.5 \mathrm{~m}$. En frecuencia de $12.5 \mathrm{~Hz}$, el error relativo es aproximadamente de $20 \%$, se presenta resonancia con la frecuencia excitadora de la plataforma $\left(f_{0}=12.5 \mathrm{~Hz}\right)$, y representa la efectividad de ambas señales $\left(X_{c}\right.$ y $\left.X_{p}\right)$, la amplitud de la respuesta de salida $\left(X_{p}\right)$ decrece en relación a la señal de comando $\left(X_{c}\right)$. En frecuencias de $15 \mathrm{~Hz}$, el sistema presenta un error relativo menor al 10\%, significa una correspondencia eficiente de la relación de ambas señales $\left(X_{c} y X_{p}\right)$, cuando el sistema es sometido a simulación por carga armónica la reproducción de la señal $\left(X_{c}\right)$ se amplifica y la respuesta de la plataforma $\left(X_{p}\right)$ responde decreciendo, sin producir mayor distorsión en el sistema. En frecuencias de $17.5 \mathrm{~Hz}$, contrariamente, la efectividad de la reproducción presenta un error relativo aproximadamente de $85 \%$, significa que la señal se relaciona con antiresonancia entre ambas señales $\left(X_{c}\right.$ y $\left.X_{p}\right)$ y el sistema se distorsiona; esta relación de la amplificación y efectividad de la reproducción de la señal.

Tabla 3: Resultados del error relativo para frecuencias de $12.5 \mathrm{~Hz}, 15 \mathrm{~Hz}$ y $17.5 \mathrm{~Hz}$.

\begin{tabular}{|c|c|c|c|}
\hline $\mathbf{X o}(\mathbf{m})$ & $\mathbf{f = 1 2 . 5} \mathbf{~ H z}$ & $\mathbf{f = 1 5} \mathbf{~ H z}$ & $\mathbf{f = 1 7 . 5 ~} \mathbf{~ H z}$ \\
\hline $\mathbf{0 . 1}$ & $12 \%$ & $12 \%$ & $77 \%$ \\
\hline $\mathbf{0 . 2}$ & $17 \%$ & $7 \%$ & $82 \%$ \\
\hline $\mathbf{0 . 3}$ & $19 \%$ & $6 \%$ & $83 \%$ \\
\hline $\mathbf{0 . 4}$ & $20 \%$ & $5 \%$ & $84 \%$ \\
\hline $\mathbf{0 . 5}$ & $20 \%$ & $4 \%$ & $85 \%$ \\
\hline
\end{tabular}

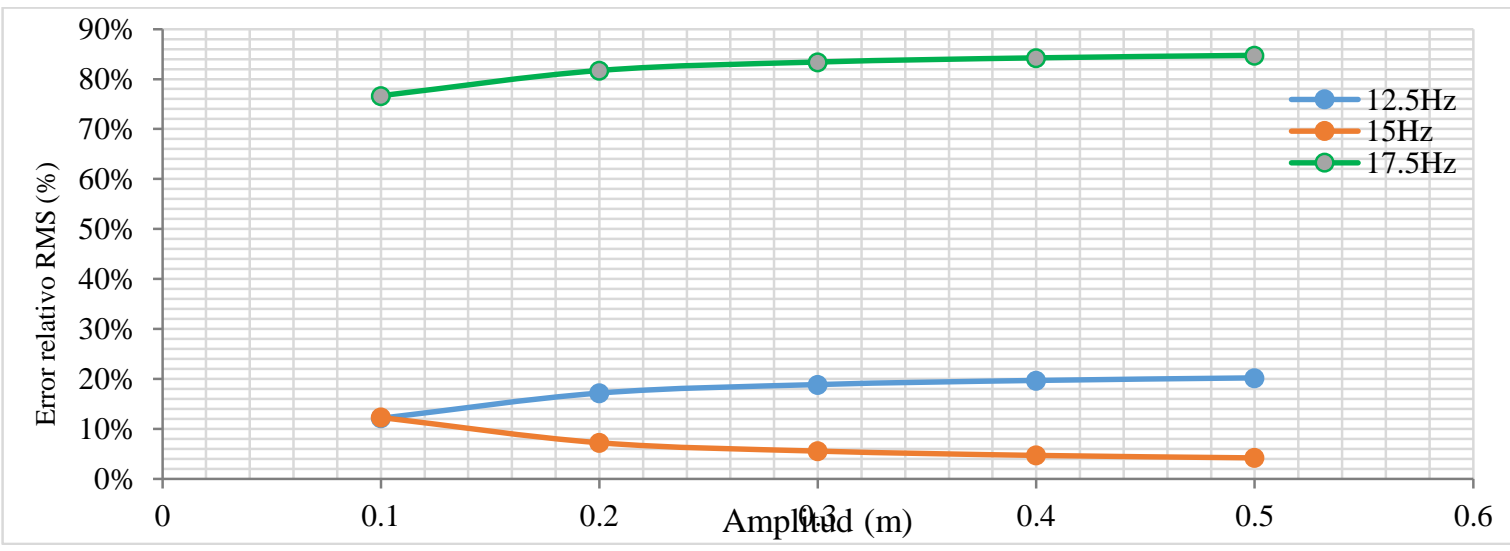

Figura 2- Error relativo para $\mathrm{f}=12.5 \mathrm{~Hz}, 15 \mathrm{~Hz}$ y $17.5 \mathrm{~Hz}$, para amplitudes de $0-0.5 \mathrm{~m}$. Fuente: Implementación en MATLAB - Simulink. 
En la tabla 4, se presenta los resultados obtenidos del estudio del error relativo medido con la raíz media cuadrática (RMS), para determinar la fidelidad en la reproducción de la señal de comando $\left(X_{c}\right)$ y la respuesta de la plataforma $\left(X_{p}\right)$, se tomó los datos para frecuencias de $20 \mathrm{~Hz}, 22.5 \mathrm{~Hz}$ y $25 \mathrm{~Hz}$, con entrada de amplitudes para la señal de comando $\left(x_{0}\right)$ de $0.1 \mathrm{~m}, 0.2 \mathrm{~m}, 0.3 \mathrm{~m}, 0.4 \mathrm{~m}$ y $0.5 \mathrm{~m}$. En frecuencia de $20 \mathrm{~Hz}$, el error relativo es aproximadamente de $75 \%$, aquí el sistema presenta distorsión, ambas señales $\left(X_{c} y X_{p}\right)$, no guardan correspondencia en la amplificación de la señal de simulación, esta relación se manifiesta en la función de transferencia, para el sistema implementado en MATLAB- Simulink. En frecuencias de $22.5 \mathrm{~Hz}$, de manera simular al anterior, el sistema se distorsiona, con un error relativo mayor al $85 \%$, la respuesta de salida $\left(X_{p}\right)$, se amplifica o decrece en relación con la señal de comando $\left(X_{c}\right)$, en esta zona el sistema indica que el control por retroalimentación de fuerzas y desplazamiento, no devuelve fidelidad en la reproducción de la señal de simulación. En frecuencias de $25 \mathrm{~Hz}$, presenta error relativo aproximadamente de $85 \%$, situación similar a la ocurrencia a partir de frecuencias de $20 \mathrm{~Hz}$; esta relación de la amplificación y efectividad de la reproducción de la señal.

Tabla 4: Resultados del error relativo para frecuencias de $20 \mathrm{~Hz}, 22.5 \mathrm{~Hz}$ y $25 \mathrm{~Hz}$.

\begin{tabular}{|c|c|c|c|}
\hline $\mathbf{X o}(\mathbf{m})$ & $\mathbf{f = 2 0} \mathbf{~ H z}$ & $\mathbf{f =} \mathbf{2 2 . 5} \mathbf{~ H z}$ & $\mathbf{f = \mathbf { 2 5 }} \mathbf{~ H z}$ \\
\hline $\mathbf{0 . 1}$ & $68 \%$ & $92 \%$ & $77 \%$ \\
\hline $\mathbf{0 . 2}$ & $73 \%$ & $87 \%$ & $82 \%$ \\
\hline $\mathbf{0 . 3}$ & $74 \%$ & $85 \%$ & $83 \%$ \\
\hline $\mathbf{0 . 4}$ & $75 \%$ & $84 \%$ & $84 \%$ \\
\hline $\mathbf{0 . 5}$ & $76 \%$ & $84 \%$ & $85 \%$ \\
\hline
\end{tabular}

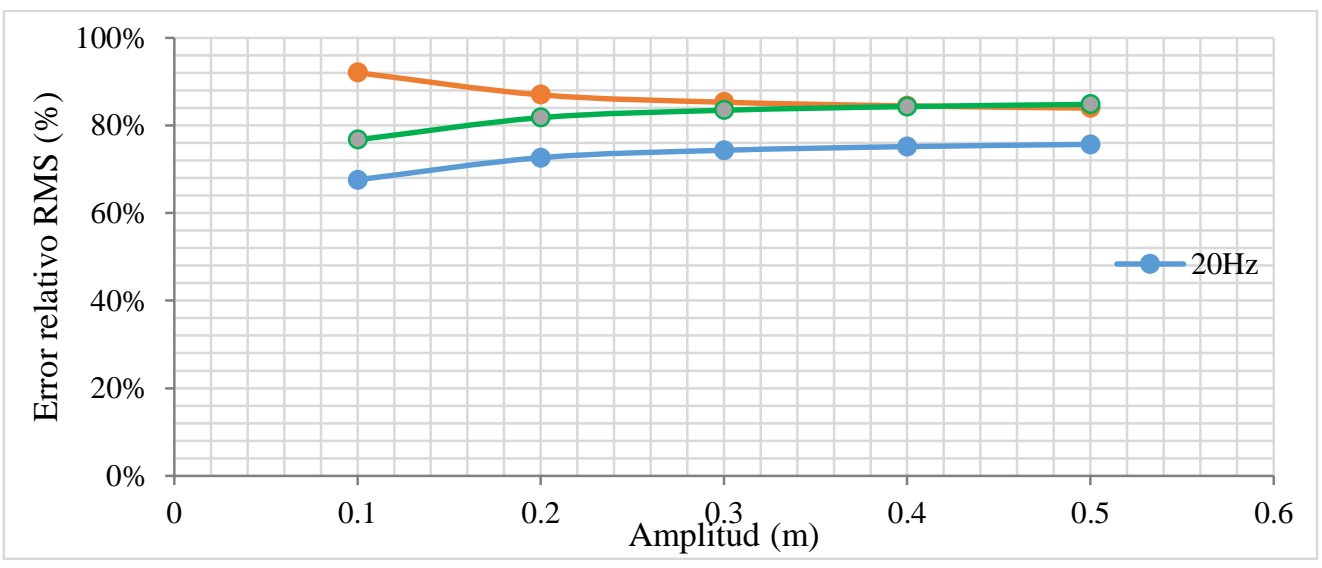

Figura 3- Error relativo para $\mathrm{f}=20 \mathrm{~Hz}, 22.5 \mathrm{~Hz}$ y $25 \mathrm{~Hz}$, para amplitudes de $0-0.5 \mathrm{~m}$. Fuente: Implementación en MATLAB - Simulink en Figura 35.

\section{Identificación con una estructura de ensayo de concreto celular de $\mathbf{2}$ grados de libertad}

En el presente ítem, estudiaremos la implementación del modelo construido en MATLAB Simulink para una mesa cargada con una estructura de ensayo de concreto celular de 2 grados de libertad, este proceso consiste en realizar un proceso iterativo cambiando las propiedades del sistema hasta que las respuestas teórica y experimental sean similares para determinar las propiedades de masa, rigidez y amortiguamiento, del pórtico en estudio. Primero, se describe a la estructura de ensayo de concreto celular, determinando la masa experimental del pórtico que se someteré a simulación sísmica en el modelo construido en MATLAB - Simulink. Seguidamente, se describe el procedimiento de construcción del modelo mesa-estructura de 2 grados de libertad en MATLAB - Simulink. Asimismo, el 
modelo se valida con carga armónica, se comprueba su error relativo y luego se somete a simulación sísmica de señales conocidas. Finalmente, se comparan los datos experimentales versus los calculados, para luego, con las respuestas máximas, determinar la masa, rigidez y amortiguamiento de la estructura de ensayo, que deberán ser similares a los datos de entrada, en el modelo analítico, demostrándose la fidelidad y eficiencia del sistema. El terreno del proyecto en mención se ubica en la Asoc. "La Agronómica" S/N del distrito Coronel Gregorio Albarracín, provincia y departamento de Tacna.

Tabla 5: Propiedades mecánicas del concreto celular

\begin{tabular}{|l|c|}
\hline \multicolumn{1}{|c|}{ Descripción } & $\begin{array}{c}\text { Vivienda de concreto } \\
\text { celular }\end{array}$ \\
\hline Peso específico $\left(\mathrm{kg} / \mathrm{m}^{3}\right)$ & 1855 \\
Resistencia, $\mathrm{f}^{\prime} \mathrm{c}\left(\mathrm{kg} / \mathrm{cm}^{2}\right)$ & 210 \\
Módulo de elasticidad, $\mathrm{E}\left(\mathrm{kg} / \mathrm{cm}^{2}\right)$ & 179551 \\
Módulo de Poisson, $\mu$ & 0,20 \\
Módulo de corte, $\mathrm{Gc}\left(\mathrm{kg} / \mathrm{cm}^{2}\right)$ & 74195 \\
Resistencia a tracción, $\mathrm{fct}\left(\mathrm{kg} / \mathrm{cm}^{2}\right)$ & 21,15 \\
Resistencia al corte del concreto $(\mathrm{kg})$ & $V c=0.53 x \frac{f c t}{1.77} \times b x d$ \\
& 0,000009 \\
\hline
\end{tabular}

Fuente: Resultados obtenidos de laboratorio de Empresa Concretos Celulares.

Se construye en el entorno de MATLAB - Simulink, el sub sistema de la estructura de 2 grados de libertad, cambiando las propiedades de la estructura, que para este caso se ingresan los datos para los dos grados de la estructura de ensayo en estudio, las variables que aparecen, son referentes a las propiedades de la estructura, son las siguientes:

- Masa de la estructura de ensayo del primer nivel $\left(m_{1}\right)$

- Masa de la estructura de ensayo del segundo nivel $\left(m_{2}\right)$

- $\quad$ Rigidez de la estructura de ensayo del primer nivel $\left(\mathrm{K}_{1}\right)$

- $\quad$ Rigidez de la estructura de ensayo del segundo nivel $\left(K_{2}\right)$

- Razón de amortiguamiento de la estructura de ensayo del primer nivel $\left(c_{1}\right)$

- Razón de amortiguamiento de la estructura de ensayo del segundo nivel $\left(c_{2}\right)$

- Respuesta de desplazamiento del primer nivel $\left(\mathrm{X}_{1}\right)$

- Respuesta de desplazamiento del segundo nivel $\left(X_{2}\right)$

Se tienen dos respuestas de desplazamiento, las correspondientes para cada nivel, estas acciones $\left(X_{1}\right.$ y $\left.X_{2}\right)$ deben ser implementadas con la respuesta de la plataforma $\left(X_{p}\right)$. Asimismo, se puede estudiar su acción en el rango de velocidades y aceleraciones para frecuencias del orden de $5 \mathrm{~Hz}$ a $20 \mathrm{~Hz}$.

\section{Función de Transferencia de la mesa cargada con estructura de 2 grados de libertad}

Para frecuencia de $5 \mathrm{~Hz}$, se presenta una zona de antiresonancia, la función de transferencia crece y decrece en la misma frecuencia, luego hasta los $12.5 \mathrm{~Hz}$, el comportamiento de ambas señales $\left(X_{c} y X_{p}\right)$ están influenciados por la acción de la frecuencia resonante de la mesa $\left(f_{0}=12.5 \mathrm{~Hz}\right)$. Sin embargo, para frecuencias superiores a $f_{0}$, la función de transferencia es influenciada por las propiedades de la estructura, primero actúa la acción de la frecuencia del primer nivel $\left(f_{1}\right)$, seguidamente la señal decrece con la influencia de la frecuencia del segundo nivel $\left(f_{2}\right)$, dicho comportamiento se debe al efecto de 
interacción dinámica de la mesa vibratoria con la estructura de ensayo, que reacciona y distorsiona el efecto de simulación con el primer nivel, amplificando la señal para los pisos superiores.

Quiere decir que, la respuesta de desplazamiento del segundo nivel $\left(\mathrm{X}_{2}\right)$ es mayor que la del primer nivel $\left(X_{1}\right)$, ambas respuestas se amplifican a la señal de comando $\left(X_{0}\right)$, y se obtiene la respuesta de desplazamiento de la mesa $\left(X_{p}\right)$.

\section{Error relativo del sistema propuesto}

En la tabla 6, se muestra el error relativo calculado mediante la Raíz media cuadrática (RMS), tema que se discutió en el ítem 4.4, con la finalidad de comparar la referencia de la amplitud de la señal de comando $\left(\mathrm{x}_{0}\right)$, con la respuesta de la mesa $\left(\mathrm{X}_{\mathrm{p}}\right)$ sometida a un sistema de control por realimentación por fuerzas y desplazamientos. Se estudió para tres frecuencias, de $5 \mathrm{~Hz}, 12.5 \mathrm{~Hz}$ y $20 \mathrm{~Hz}$, zonas donde la función de transferencia presenta transición de señal, que representa puntos de quiebre en la fidelidad de la reproducción durante la simulación sísmica.

Sin embargo, el modelo implementado para una mesa cargada con estructura de ensayo de 2 grados de libertad, presenta un error relativo menor en promedio de $3.5 \%$. Lo que significa que el sistema implementado, puede reproducir la señal de comando en frecuencias altas, para lo cual se tomará dicho modelo, en la identificación de los parámetros de la estructura de ensayo. La estructura de ensayo propuesta, se desarrolló con las propiedades del concreto celular, material de propiedades mecánicas menores a la del concreto convencional, quiere decir, que, durante la simulación, el resultado de las respuestas experimentales versus las calculadas, nos permitirán encontrar su masa, rigidez y amortiguamiento, además de evaluar la influencia de estos, sobre el sistema.

Tabla 6: Resultados del error relativo para frecuencias de $5 \mathrm{~Hz}, 12.5 \mathrm{~Hz}$ y $20 \mathrm{~Hz}$.

\begin{tabular}{|c|c|c|c|}
\hline $\mathbf{X o}(\mathbf{m})$ & $\mathbf{f = \mathbf { 5 ~ H z }}$ & $\mathbf{f}=\mathbf{1 2 . 5} \mathbf{~ H z}$ & $\mathbf{f = \mathbf { 2 0 }} \mathbf{~ H z}$ \\
\hline $\mathbf{0 . 1}$ & $5.3 \%$ & $4.9 \%$ & $4.5 \%$ \\
\hline $\mathbf{0 . 2}$ & $4.6 \%$ & $4.2 \%$ & $3.8 \%$ \\
\hline $\mathbf{0 . 3}$ & $3.2 \%$ & $2.8 \%$ & $2.4 \%$ \\
\hline $\mathbf{0 . 4}$ & $2.8 \%$ & $2.4 \%$ & $2.0 \%$ \\
\hline $\mathbf{0 . 5}$ & $2.5 \%$ & $2.1 \%$ & $1.7 \%$ \\
\hline
\end{tabular}

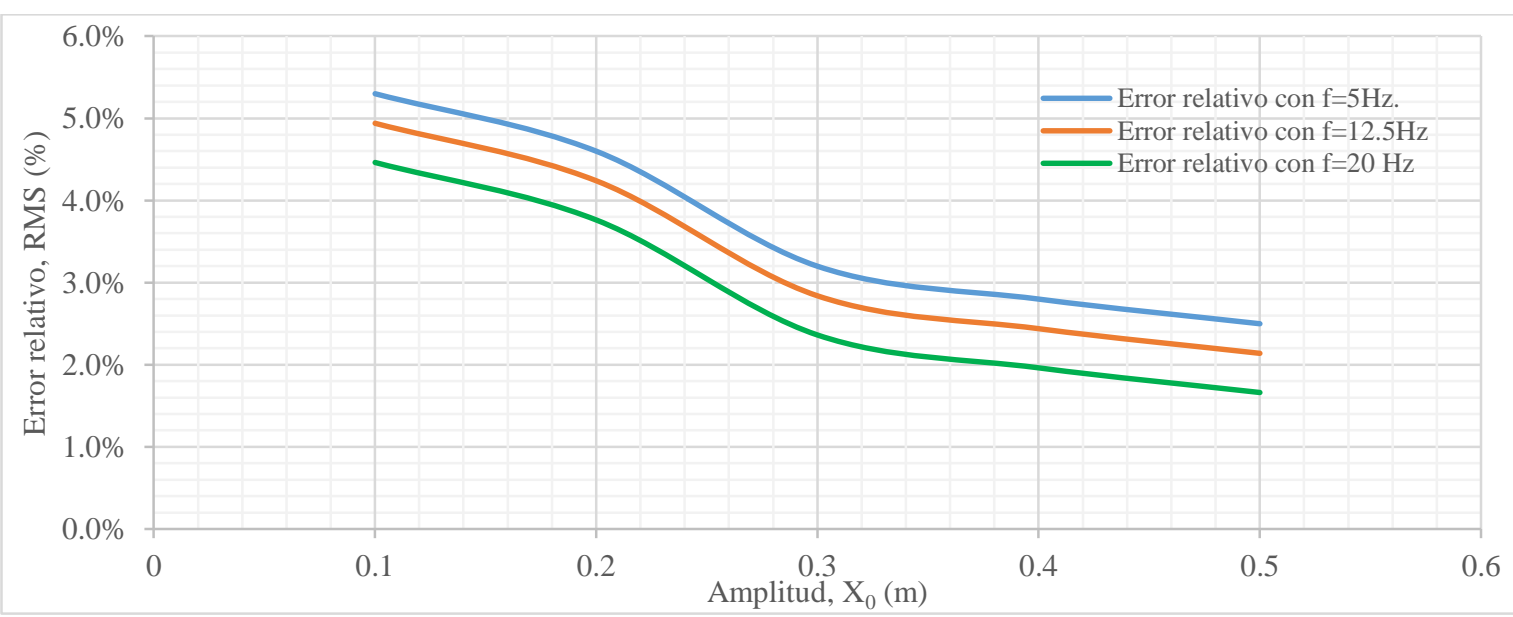

Figura 4- Error relativo para $\mathrm{f}=5 \mathrm{~Hz}, 12.5 \mathrm{~Hz}$ y $20 \mathrm{~Hz}$, para amplitudes de $0.1 \mathrm{~m}$ a $0.5 \mathrm{~m}$. Fuente: Implementación en MATLAB - Simulink 
Según las características de la estructura de ensayo, se trata de una estructura simétrica, que tiene masas constantes en cada pórtico principal, por ello analizaremos un pórtico típico. Para identificar los parámetros de la estructura de ensayo, sometemos el sistema a dos registros de aceleraciones para validar el comportamiento de la estructura de ensayo y comparar sus propiedades (masa. Rigidez y razón de amortiguamiento), del ensayo experimental con el valor calculado, demostrando la fidelidad del sistema.

\section{DISCUSIÓN}

En lo referente al tema de estudio creemos que la principal aportación que este proyecto puede hacer, es destacar los criterios que en la implementación de un controlador para señales armónica y sísmica propuestos por Esparza (1986), para lo cual se representó en MATLAB - Simulink, el modelo analítico con ayuda de los diagramas de bloques que ofrece esta aplicación, con el objetivo de obtener las características de la respuesta, con retroalimentación de fuerzas y desplazamientos, una vez validado el modelo experimental para evaluar la interacción de la mesa plataforma con estructura, se propone analizar una estructura con propiedades de concreto celular, mediante la identificación de sus parámetros (masa, amortiguamiento y rigidez). Con la finalidad de estudiar la influencia de estos parámetros en el comportamiento dinámico, comparando las respuestas del estudio experimental y calculado, aplicando el principio de error relativo con RMS, se concluyó que el modelo diagramado en MATLAB - Simulink sometido a una señal sísmica, demuestra eficiencia en los resultados evaluados para sistemas con retroalimentación de fuerzas y desplazamientos.

El sistema de compensación en MATLAB - Simulink contribuye con la fidelidad de la reproducción del movimiento, controlando la resonancia excesiva, además permiten la coincidencia entre la amplitud de la señal de comando y el desplazamiento de la plataforma., esto se logró determinar con el cálculo del error relativo - RMS para diferentes frecuencias y con la aplicación de 2 registros de aceleraciones sísmicas,

La función de transferencia propuesta por Esparza (1986), es representada en MATLAB - Simulink reproduciendo fielmente la señal sísmica para sistemas de 2 grados de libertad, esto determina que cuando la estructura de ensayo de concreto celular recibe la fuerza, su comportamiento ante los ensayos de simulación sísmica nos permite reflejar con fidelidad dichas señales, además, al someter al sistema ante impulsos de alta frecuencia a los cuales denominamos "ruido", el sistema no se perturba y la propuesta de retroalimentación por fuerza y desplazamiento, demuestra eficacia en las respuestas del sistema actuador mesa - estructura.

Del análisis en MATLAB - Simulink, se presentan los resultados experimentales versus los calculados, donde se evidencia una correspondencia racional de la respuesta de desplazamiento y velocidades, ante una señal sísmica; además, la relación de masas (u) también, es un parámetro de estudio, debido a que el sistema validado en MATLAB - Simulink se propone para una relación inferior a 1.0, con lo que se demuestra la fidelidad en la reproducción de la señal sísmica, sin embargo, el haber escogido un material de propiedades mecánicas inferiores a las del concreto convencional, debería suponer un mayor control en la aplicación de la señal sísmica, pero se demuestra que con este tipo de material y cualquier otro tipo, la representación del modelo representa un avance muy importante en el estudio de la ingeniería sismoresistente, que evalúa el comportamiento del sistema como un sistema dual (mesa - estructura) en sus diferentes respuestas (aceleración, velocidad, desplazamiento) con la finalidad de atenuar dichas respuestas en razón de las propiedades dinámicas del sistema propuesto.

La estructura de ensayo propuesta contiene propiedades de concreto celular, lo que significa estudiar una estructura con alta capacidad de amortiguamiento, lo que da como respuesta evitar excesivas resonancias y atenuaciones, y con ello es posible incrementar el grado de estabilidad, por lo 
tanto, esta amplificación por resonancia tiene tendencia a disminuir de magnitud al aumentar el valor de la frecuencia natural de la estructura. Quiere decir que a mayor frecuencia de la estructura le corresponde mayor distorsión en antiresonancia. Donde, el error relativo se determinó en el modelo construido en MATLAB - Simulink con el bloque RMS para el análisis experimental con la estructura de ensayo, dando como resultado un error inferior al 3.5\%, que en estadística para el rango de valores es aceptable, con ello los datos calculados versus los experimentales, permiten identificar los parámetros de masa, amortiguamiento y rigidez con los que se comporta una estructura de concreto celular al interactuar con un efecto externo (señal sísmica), en un primer instante la diferencia entre ambos resultados es mayor pero en su trayectoria se estabiliza, para un sistema actuador mediante retroalimentación de fuerzas y desplazamientos.

Se concluye que, con el estudio de interacción entre mesa vibratoria y estructura de ensayo con propiedades de concreto celular, del modelo construido en MATLAB - Simulink, en el que se incluyó "ruido" - perturbaciones de alta frecuencia, al realizar el estudio del error relativo -RMS de la respuesta se tiene como resultado que para frecuencias de $15 \mathrm{~Hz}$, superior a la frecuencia de la plataforma, se disminuye el error en valores menores al $10 \%$, evidenciándose una disminución en la actuación de las perturbaciones sobre el sistema. El modelo construido en MATLAB - Simulink, responde eficientemente en la estabilización del sistema al ser sometido a una simulación sísmica, además participa la relación de masas donde $\mathrm{u}=0.5$ evidencia mayor aproximación entre ambos modelos. El modelo analítico, significa un mayor estudio en los requisitos de estabilidad del sistema y de la fidelidad en reproducir la señal de comando. Por lo tanto, es necesario detallar adecuadamente ambos requisitos, durante el desempeño del modelo propuesto y el espécimen de ensayo. En lo referente, a la identificación de parámetros, con el modelo validado en MATLAB - Simulink, se ensayó para una estructura de concreto celular de dos niveles, con lo cual se tuvo resultados experimentales versus los calculados, comprobándose la fidelidad del sistema, al reproducir una señal sísmica uniaxial, sin embargo las razones de amortiguamiento calculadas versus la experimental tiene una menor aproximación en los resultados, considerando que los datos de entrada para el método calculado, presume una relación de masas del orden $u=0.50$, quiere decir que nuestra estructura de características livianas, tuvo un error relativo - RMS inferior al 3.5\%, esto indica que debería actuar de manera similar en cualquier otro tipo de estructura, manejando la relación antes mencionada, entonces, con ello se concluye que el sistema actuador mesa-plataforma debe ser utilizado para evaluar cualquier tipo de material en estructura, controlando las distorsiones y validando la correspondencia de la realimentación de fuerzas y controlado por desplazamientos.

El presente trabajo de investigación, debe profundizarse, en ampliar el modelo actuador plataforma - estructura, para estructuras de $\mathrm{n}$ grados de libertad, así como el estudio de otros sistemas de control, para encontrar la fidelidad en la reproducción de las señales sísmicas, que nos den una mejor aproximación de cómo se comportará la estructura ante un evento sísmico real de gran magnitud.

\section{REFERENCIAS BIBLIOGRÁFICAS}

Aracil, Javier \& Gómez - Estern, Fabio. (2007). Introducción a Matlab y Simulink, Universidad de Sevilla. España.

Bernal, Mauricio, Aponte, Jorge \& Carrillo, Julián. (2015). "Sistemas de control para mesas vibratorias: una revisión crítica", Universidad Militar Nueva Granada, Colombia.

Blondet, M \& Esparza, C. (1988). Analysis of Shaking table-structure interaction effects during seismic simulation tests. Earthquake Engineering and Structural Dynamics, Vol. 16, 473-490.

Blondet, M \& Velásquez, J. (2016). Sistema de control para una mesa vibratoria de 3 grados de libertad durante ensayos de simulación sísmica. Lima, Perú. WECDRR 2016, pp. 1-10. 
Bonilla Sosa, Lily Marlene (2012). Teoría del aislamiento sísmico para edificaciones. Universidad Nacional Autónoma de México.

Dorf, Richard C. (1983). "Modern Control Systems"; Addison-Wesley Publishing Company, Reading, Massachusetts.

Esparza Díaz, Carlos Alfredo (1986). Efectos de interacción estructura - mesa vibradora durante ensayos de simulación sísmica. PUCP - Lima, Perú, pp. 1-174.

Gaviño Hernández Ricardo (2010). Introducción a los sistemas de control: Conceptos, aplicaciones y simulación con MATLAB. Prentice Hall.

Harrison, Howard L. \& John G. Bollinger. (1983).“Controles Automáticos”; Editorial Trillas, México

Norma Técnica E.030 “Diseño Sismorresistente" (2006). Aprobada mediante Decreto Supremo № 0032016-VIVIENDA, Lima. Perú.

Ogata, Katsuhiko (1978). "Systems Dynamics"; Prentice-Hall, Inc., Englewood Cliffs, New Jersey.

Ogata, Katsuhiko (1974). "Ingeniería de Control Moderna"; Editorial Prentice -Halla Internacional, Madrid 1974.

Popov, E.P.(1962). "The Dynamics of Automatic Control Systems"; Addison-Wesley Puslishing Company, Reading, Massachusetts.

Yang, Kang Li, T.Y., Jian-Yuan Lin, Yuanjie LI, D.P. Tung, \& J. Fawcet (2017). "Advanced shake table controler design using model predictive control strategy", paper publicado en Berkeley, California.

Yasundo Takahashi, Michael J. Rabins \& David M. Auslander. (1970). "Control and Dynamics Systems"; Addison-Wesley Publishing Company, Berkeley, California. 